\title{
Investigation of Secondary Hardening in MP35N Wires
}

\author{
D. Sorensen ${ }^{1,2}$, B.Q. Li $^{1}$, W.W. Gerberich ${ }^{2}$, and K.A. Mkhoyan ${ }^{2}$ \\ ${ }^{1}$ Medtronic Neuromodulation, Fridley, Minnesota 55432 \\ ${ }^{2}$ Chemical Engineering and Materials Science, University of Minnesota, Minneapolis, MN 55455
}

MP35N (Co-35Ni-20Cr-10Mo) is a superalloy used in cardiac pacing and neurostimulation lead conductor applications. When fine MP35N wires are exposed to elevated temperatures for brief periods of time during secondary processing steps such as wire insulation, stress relief, and cabling the strength increases of $25 \%$ are observed. The mechanism of secondary hardening has been previously studied and the results have been controversial. Precipitation of $\mathrm{Co}_{3} \mathrm{Mo}$ [1], thickening of hexagonal close packed (HCP) phase platelets [2], and Suzuki segregation [3] have all been proposed as possible strengthening mechanisms.

In this study, annular dark field - scanning transmission electron microscopy (ADF-STEM), energy dispersive $x$-ray spectroscopy (EDS), energy filtered TEM (EFTEM), and electron backscatter diffraction (EBSD) was used to investigate the local chemistry at microstructural defects. Results of the microstructural characterization were correlated with mechanical test data on fine MP35N wires.

Tension testing MP35N in the as drawn and as-drawn followed by aging for 60 seconds at temperatures ranging from $300^{\circ} \mathrm{C}$ to $1000^{\circ} \mathrm{C}$ showed a gradual increase in yield stress reaching a maximum at $700^{\circ} \mathrm{C}$. Exposing MP35N wires to elevated temperatures for short durations is common while processing medical devices and while these processes strengthen the material, large amounts of ductility are lost depending on the prior dislocation density of the material. Understanding the mechanism for the unique secondary hardening effects in MP35N fine wires is necessary for robust design, lean processing, and product reliability in the demanding in-vivo environment medical devices are exposed to.

Electron backscatter diffraction was used to characterize the evolution of crystallographic orientation, grain size, and grain boundary character as a function of aging temperature. The EBSD analysis showed no significant microstructure differences until the onset of recrystallization. Conventional transmission electron microscopy (CTEM) and selected area diffraction (SAD) was used to image the microstructure of MP35N and identify second phases if present. TEM imaging and SAD analysis of aged MP35N showed microstructures consisting of deformation twins, stacking faults, and dislocations; all consistent with previous [3] and current investigations.

STEM and EDS were used to probe the microstructure for local chemistry changes at defects. ADFSTEM imaging showed increased contrast at stacking faults and microtwins (Figure 1) while EDS line scans revealed increased concentration of molybdenum at the stacking faults (Figure 2). These findings show Suzuki segregation in MP35N.

\section{References}

1. Raghavan, M., B.J. Berkowitz, and R.D. Kane, Metall. Mater. Trans. A 11A, 203 (1980).

2. Singh, R. and R. Doherty, Metall. Mater. Trans. A 23(1), 307 (1992).

3. Asgari, S., et al., Acta Mater. 46(16), 5795 (1998). 

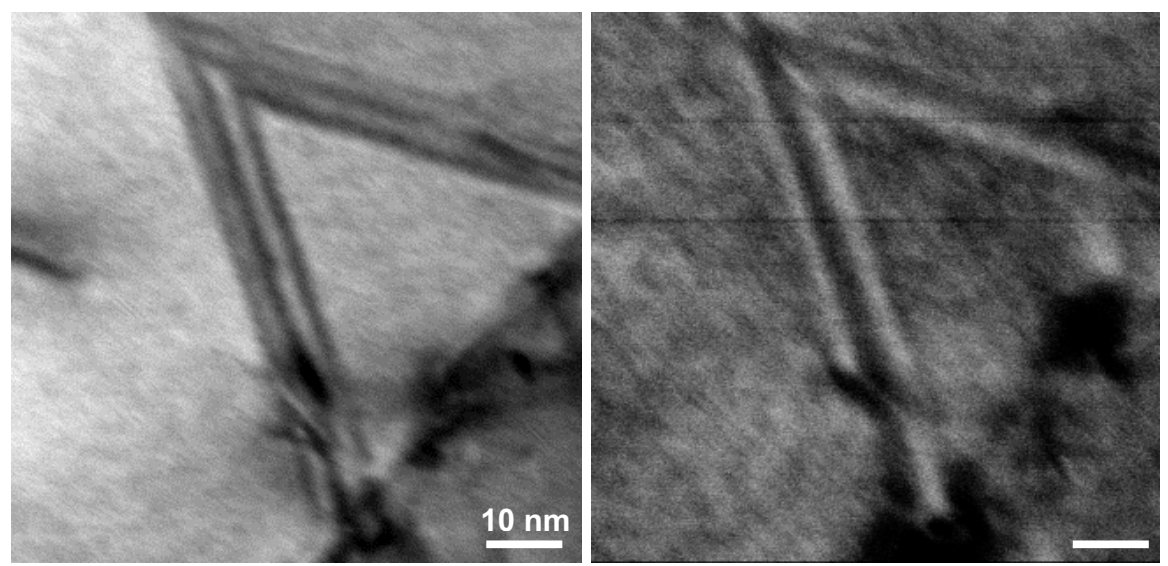

Figure 1. (a) BF and (b) high angle ADF STEM images of stacking faults in MP35N rod aged at $600^{\circ} \mathrm{C}$ for $30 \mathrm{~min}$.
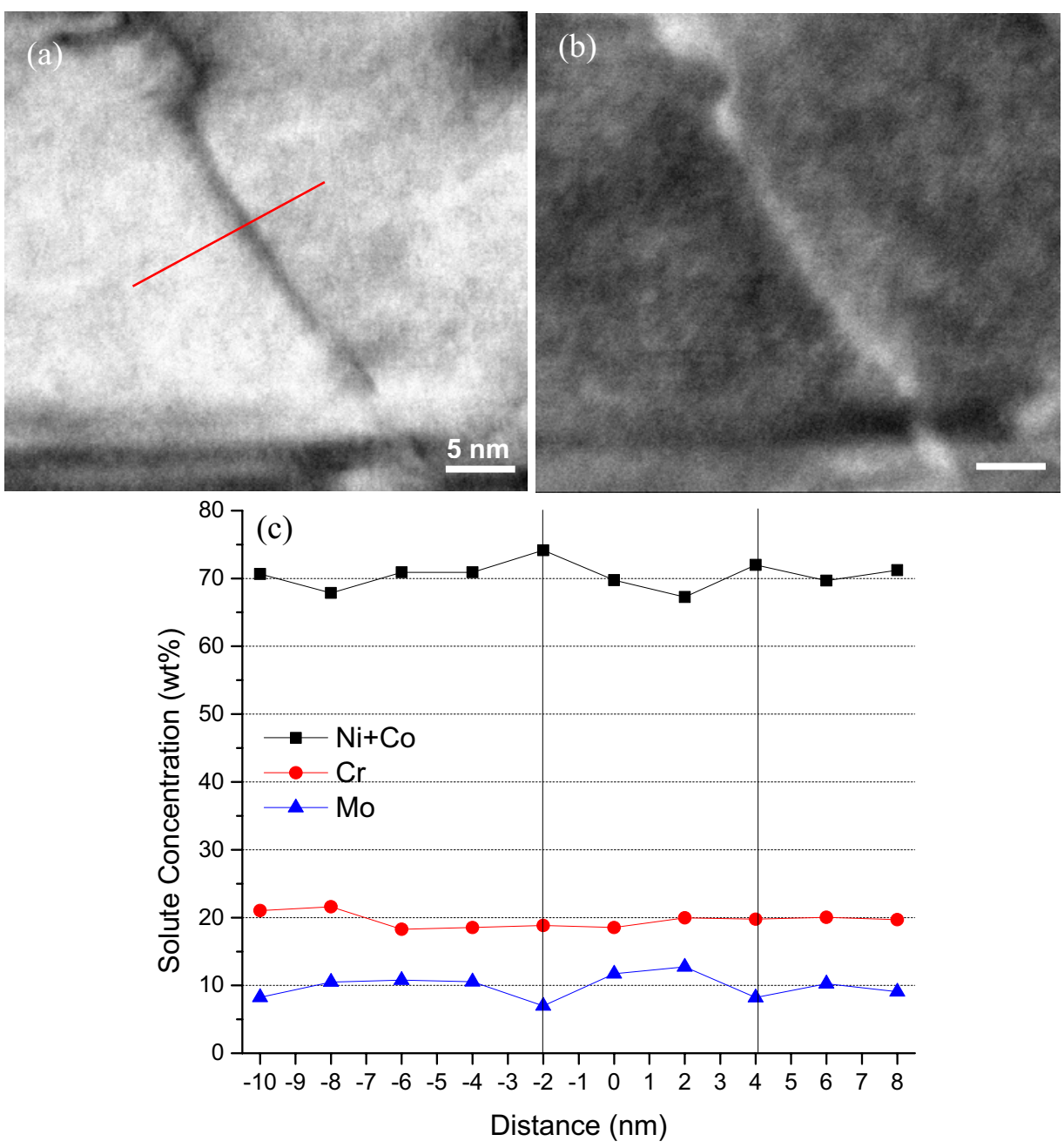

Figure 2. (a) BF STEM image showing location of EDS line scan across an edge on stacking fault. (b) HAADF STEM image from the same stacking fault. (c) Concentration of elements across the stacking fault measured using EDS (shown in (a)). Mo rich region on and near the fault can be observed. The area adjacent to the fault is poor in Mo and rich in $\mathrm{Ni}$ and $\mathrm{Co}$. 